\title{
MIR377 Pre-miRNA
}

National Cancer Institute

\section{Source}

National Cancer Institute. MIR377 Pre-miRNA. NCI Thesaurus. Code C82829.

MIR377 is an oligoribonucleotide that is encoded by the human MIR377 gene and has a role in the regulation of gene expression. 